\title{
Microbial Community Shift and Role of Bacteria in Rapid Granulation By Using Diatomite
}

\author{
Aznah Nor Anuar \\ Universiti Teknologi Malaysia \\ Mohd Hakim Abdul Halim \\ Universiti Teknologi Malaysia
}

Hazlami Fikri Basri ( $\nabla$ hazlami@utm.my)

Universiti Teknologi Malaysia - Main Campus Skudai: Universiti Teknologi Malaysia

\section{Research}

Keywords: Aerobic granular sludge, Diatomite, Metagenomic analysis, Microbial community

Posted Date: January 4th, 2022

DOI: https://doi.org/10.21203/rs.3.rs-1099681/v1

License: (1) This work is licensed under a Creative Commons Attribution 4.0 International License.

Read Full License 


\section{Abstract}

Aerobic granular sludge (AGS) technology is a promising biological method for modern wastewater treatment. However, granulation time have become a major issue for the application of AGS technology especially in low strength wastewater. Recent studies on granulation are focusing towards rapid start-up granulation process. Diatomite, a friable light-coloured sedimentary rock was introduces in this study to enhanced the granules formation. This study highlight the effect of diatomite towards the microbial community during the transformation of seed sludge until development of granules. DNA extraction and Metagenomic analysis was conducted with three samples (seed sludge, control AGS, AGS diatomite) to compare the microbial community. The microbial community analysis revealed the alpha diversity, phylum and class level, and the abundance of EPS producing bacteria of each bacteria samples respectively. Diatomite has a significant influence towards the microbial diversity (High Shannon index alpha diversity). Also, diatomite promotes the abundance of functional bacteria especially EPS producing bacteria, which seen as a crucial elements in granulation process.

\section{Introduction}

Modern technology has introduced more advance system in both biological and chemical method for wastewater treatment process. Nowadays, biological treatment becomes more preferable than additional of chemicals which provides more cost effective, simple and environmentally friendly system for the treatment [1]. In the need for more compact biological treatment, there has been an evolution in the sludge discovery for the application of wastewater treatment. Aerobic granular sludge (AGS) technology is seen as a preferable alternative than using activated sludge system in treating wastewater. AGS technology demonstrates a $20-25 \%$ reduction in operation costs, $23-40 \%$ less electricity requirement and a $50-75 \%$ reduction in space requirements [2]. Aerobic granular sludge is widely recognized as one of the promising technologies for modern wastewater treatment. A lot of studies on AGS have been done for the past decades and even scaled-up to full-scale reactors which are currently operational in the Netherlands, Portugal, and South Africa [3]. Aerobic granular sludge was regarded by some researchers as a suspended spherical biofilm that included microbial cells, inert particle, degradable particles, and extracellular polymeric substances (EPS) [4].

Microbial diversity is a crucial element that play a major role on influencing biological wastewater treatment. The emergence of bio granulation technology with high biological treatment quality is reported to be correlated with microbial diversity. Many researchers discovered that aerobic granules developed in bio granulation technology have a greater microbial diversity compared to conventional activated sludge $[5,6]$. It was revealed that each species of microbes in the aerobic granules performed different roles in the formation of AGS and removal efficiencies of the treatment $[7,8,9,10,11]$. For the last decades, many researchers have been working to investigate the method to enhance the granulation process. Different operational strategies were used and they result in different granules characteristic and performances. For instances, shortening the settling time [12] increased Food/ Mass (F/M) ratio [13] and also increased

organic loading rate [14]. All of them successfully formed AGS in short period of time, however they were 
only applicable to high strength wastewater as the substrates. Low strength domestic wastewater was still a major concern for bio granulation technology. This condition led to the microbial diversity analysis in the need to discover the granules inner component.

Fundamentally, the aerobic granulation is a novel progressive evolution of dispersed seed sludge into a solid dense granule. The granules consist of dense microbial consortia packed with different bacterial species. A suitable environment was crucial for the interaction between microbes especially at the initial stage of the granulation process. Diatomite, one of the few support materials has drawn considerable attention among researchers for the past few years. Diatomite is a friable light-coloured sedimentary rock formed from naturally occurring fossilized remains of diatoms. It is made up of shells of single diatoms and readily crumbles into a fine powder [16]. In general, diatomite quality was highlighted by the overall characteristic; resourceful, economical friendly, and high adsorption quality [16]. In the bio granulation perspective, diatomite has a unique characteristic that makes it relevant to enhance the formation of AGS. Diatomite was seen as a good carrier that functions as a 'home' which provide a convenient environment for the microbes to grow. Moreover, high porosity and large surface area make the diatomite capture the suspended microbes quickly [15]. This could promote the accumulation of microbes and lead to rapid granular formation. In this study, diatomite was used as a catalyst for granulation process. Therefore, the evolutionary shift of microbial diversity during the development of granules with the presence of diatomite was highlighted in this study.

\section{Methodology}

\subsection{Bioreactor Set-Up and Operation}

The sample of real domestic wastewater was obtained from Bunus Sewage Treatment Plant (STP), Kuala Lumpur, Malaysia. The COD concentration was $184 \pm 15.4 \mathrm{mgL}^{-1}$ which is considered less than $200 \mathrm{mgL}^{-}$

${ }^{1}$. Sguanci et al. [17] stated that wastewater with COD concentration lower than $200 \mathrm{mgL}^{-1}$ was categorized in low strength wastewater. The accompanied parameter such as $\mathrm{NH}_{3}-\mathrm{N}, \mathrm{TIN}$, and $\mathrm{PO}_{4}-\mathrm{P}$ were all recorded higher concentration than the standard. The range of $\mathrm{NH}_{3}-\mathrm{N}$ concentration for this study was $21.8 \pm 5 \mathrm{mgL}^{-1}$, while TIN and $\mathrm{PO}_{4}-\mathrm{P}$ concentrations were $63.5 \pm 8 \mathrm{mgL}^{-1}$ and $13.30 \pm 3 \mathrm{mgL}^{-1}$ respectively. The experiments have been carried out in a solid cylindrical acrylic bioreactor, having a diameter of $17 \mathrm{~cm}$, and a height of $125 \mathrm{~cm}$, to operate with a working volume of $15 \mathrm{~L}$. The development of aerobic granules in the bioreactor was divided into two conditions. The first condition was the development of naturally formed AGS (AGS control) and the latter was development of AGS with the addition of diatomite (AGS diatomite). The only difference between them was the diatomite. At the beginning of the experiment, $7.5 \mathrm{~L}$ of activated sludge from Bunus STP was added as inoculums to fill half of the working volume of the bioreactor during the start-up period. While, $5000 \mathrm{mgL}^{-1}$ of diatomite dosage was added into the bioreactor during the early stage for the case of AGS diatomite development. The dosage was added only during the start-up period and no further addition of diatomite needed. The bioreactor operated under sequencing batch mode for 24 hours continuously with a cycle of 3 hours. The 
3-hour cycle consists of 60 min of influent feeding, $110 \mathrm{~min}$ of aeration, 5 min of sludge settling, and 5 min of effluent discharge. A feeding, discharge, and an air pump with the setting time for each phase in the bioreactor was controlled with pre-programmed digital timers. Feeding and discharge of wastewater were managed by a set of two peristaltic pumps. As for the aeration pump, it supplies oxygen through a diffuser placed at the bottom of the bioreactor with a volumetric flow rate of $0.18 \mathrm{~m} 3 \mathrm{~h}-1\left(0.22 \mathrm{~cm} \mathrm{~s}^{-1}\right.$ superficial airflow velocity). The influent was fed into the inlet at the bottom of the bioreactor while the effluent was discharged through the outlet ports in the middle of the bioreactor. The effluent had a volumetric exchange ratio (VER) of $50 \%$ before the location of the outlet ports. The solid retention time (SRT) and Hydraulic retention time (HRT) was set according to the AGS control and AGS diatomite experiment. The bioreactor was scheduled to run a specific period without excess sludge discharge; thus, the effluent was the only passage for biomass wasting to be transported. The temperature in the bioreactor was kept at $27 \pm 1^{\circ} \mathrm{C}$. The morphological characteristic parameter, the granules' structure and microstructure were examined by using a stereomicroscope equipped with a digital image analyzer (Olympus SXZ7).

\subsection{Microbial Community in AGS}

Three different samples consist of seed sludge, controlled AGS (without diatomite) and AGS diatomite were collected from the pilot bioreactor for microbial community analysis. The seed sludge was directly taken from the aeration tank while the controlled AGS was drawn from the bioreactor after the AGS was successfully developed (after 93 days). The same case goes to AGS diatomite after it was cultivated for 50 days in the bioreactor. The three samples were subjected to DNA extraction via Macherey-nagel nucleospin soil kit. According to the manufacturer's protocol, the extracted DNA was first purified by electrophoresis where its solution was processed into a $1 \%(\mathrm{w} / \mathrm{v})$ agarose gel and the DNA was visualized under UV light. After that, the isolated DNA was evaluated via spectrophotometry to measure the concentration and for further purification using NanoDrop 1000 V3.7 Spectrophotometer

(ThermoScientific). Later, the sequencing was carried out by using lon $16 \mathrm{~S}^{\mathrm{TM}}$ Metagenomics Kit through Ion Torrent sequencing technology. Begin with PCR amplification, the lon $16 \mathrm{~S}^{\text {TM }}$ Metagenomics Kit was conducted with primer set V2-4-8 which were used to amplify the corresponding region of the 16S rRNA gene. The process going through a paired-end sequencing on one lane to generate 100 bp paired-end DNA fragment sequences in fastq format. The raw sequences contaminated by adapter or containing three or more unknown nucleotides (' $N$ ') were removed via the quality control (QC) pipeline using a modified Dynamic Trim. A phred score of 15 was taken as the high-quality base and the sequences were trimmed when the phred score was below than five. This was to ensure that all the datasets generated during the next generation sequencing were of high quality. Afterwards, the amplified fragments were sequenced on the lon $\mathrm{PGM}^{\mathrm{TM}}$ System and the results were analyzed with the lon Reporter ${ }^{\mathrm{TM}}$ software and Ion $16 \mathrm{~S}^{\mathrm{TM}}$ Metagenomics Kit analyses module. After the data is imported, the analysis results that contains the data arranged in column, rows and pie chart were presented. The visualizations of analysis results were performed in Ion Reporter ${ }^{\text {TM }}$ Software with lon Reporter ${ }^{\text {TM }}$ Genomic Viewer (IRGV).

\section{Result And Discussion}




\subsection{Formation of Biogranules}

The development of AGS diatomite is different from natural granulation process (AGS control). Both granules (AGS and AGS diatomite) were successfully formed in this study. However, it is important to highlight that the time taken to achieve granulation is the main difference between both types of granules. AGS control took a significantly longer period to become a granule. The images of AGS diatomite and AGS control at the end of experiment was shown in Figure 1. It was noted that at the beginning of the experiment, both developed granules have the same startup condition with the majority of the sludge was considered flocculent sludge size of less than $0.3 \mathrm{~mm}$. Subsequently, in just 10 days, the diatomite effect was already noticed. A huge number of small granules was observed in the bioreactor system. This rapid transition of seed sludge to granular sludge indicates the influence of diatomite functioning as a nucleation agent that attracted surrounding microbes and suspended organics to initiate granulation process. According to Sarma et al [18], the first phase of AGS formation (cell to cell interaction), normally takes a long period before entering the second phase (micro aggregate formation). This could be seen by the control AGS having a passive granulation rate since the start of the experiment. Similar transformation could only be achieved after more than 50 days of the experiment.

Diatomite plays a critical role in initiating the aggregation process during this early stage. Due to its chemical composition and microstructure, the surface of diatomite creates a Beta potential that could neutralise other particles. The beta potential enabled the particles to be adsorbed to the diatomite and agglomerated to become a floc and small granules [19]. With the achievement of initial aggregation, the subsequent granulations are easy to proceed [20]. Later, the AGS diatomite continues to develop and undergo clear morphological transformation becoming more compact and smoother surface at the 20th day. The colour of the granules was shifted into light brown as compared to previous cases (dark brown). In this stage, the numbers of developed AGS diatomite inside the bioreactor were rapidly increased. Correspondingly, the performance of the system towards the settling ability and the removal of organics and nutrients also became more efficient. Differently, AGS control going through the process in a very slow rate. Only after 60 days, the small granules started to appear in the bioreactor. Normal granulation depends highly on the microbial diversity to triggered the granules formation. In most domestic wastewater cases, low organics which interconnected with microbial diversity contribute to the slower granulation rate. Low organic loading causes low microbial diversity [21].

AGS control successfully attained granulation ( $91 \%$ granulation rate) after 85 days of bioreactor operation. The majority of the developed granules was small in diameter ranging from 0.6-1 mm. Remarkably, the feat that was achieved by AGS controlled at the end of bioreactor operation, was easily attained by AGS diatomite in less than 21 days. AGS diatomite accomplishes $92 \%$ of granulation rate at day 20. Notably, the contrast towards the aerobic granulation period for both granules was distinct with 65 days' difference. Mature diatomite granules with a bigger, compact and smooth surface were visualized on the 30th day as shown in Figure $1 \mathrm{~b}$ until the end of the experiment. In comparison to the condition of both granules on day 50, vast majority (56.7\%) of the AGS diatomite size ranging from 1 to 4 $\mathrm{mm}$ while for controlled AGS, the majority (42\%) of them was still flocculent sludge with less than 0.3 
$\mathrm{mm}$ size. Only $13.8 \%$ of the AGS control have the size of $1-4 \mathrm{~mm}$ on day 50 . This comparison highlights the immediate effect of diatomite in promoting rapid granulation compare to the natural granulation process. Prominently, the short duration of AGS diatomite formation enhanced the sustainability of the treatment and at the same time, improving the efficiency and effectiveness of the system.

\subsection{The Alpha Diversity of the Microbial Community}

High-throughput pyrosequencing of 16S rDNA amplicon technology was conducted to analyse the microbial diversity and community structures of three samples; seed sludge, controlled granules on day 90 and AGS diatomite on day 30. The alpha-diversity between the three samples were shown in Table 1. The alpha diversity indices are Ace, Chao, Shannon and Simpson. Ace and Chao represent the numbers of different taxonomy units for community richness while Shannon and Simpson indicate community diversity. The microbial richness was defined as the count of the different species present in the community whilst microbial diversity was described as the abundance or number of individuals of different species in the community. A higher value of Ace and Chao demonstrates higher richness. Additionally, higher Shannon indices highlight higher diversity while high Simpson indices demonstrate low community diversity [8]. Based on the 3 samples, all alpha diversity indices showed that seed sludge exhibited a relatively higher species richness than controlled and AGS diatomite. Referring to Table 1, the Chao index representing species richness was the highest for seed sludge, compared to both developed granules. It was possibly due to the heavy washout experienced by the biomass in both bioreactors during the experiment, and a quite simple substrate composition in the influent as compared to municipal wastewater. The reduced in species richness in granules are comparable to previous studies by $\mathrm{He}$ et al. [7], Zou et al. [22] and Jiang et al. [10]. The decreased in richness after granulation was a common occurrence due to different condition and microbial functions inside the granules. Notably, the microbial diversity was important during the early stage of granules formation to undergo cell to cell formation and lead to a faster aggregation process. Meantime, AGS diatomite recorded a higher microbial diversity than controlled and seed sludge after achieving the highest Shannon index. The difference was seemingly due to the presence of diatomite that played a decisive role in promoting the growth of microbes. This finding was comparable to Yang et al. [23] that conducted a study on the enhanced mechanism of a novel diatomite biofilm in the pretreatment process of treating polluted raw water. It was stated that the presence of diatomite would alter the accumulation characteristic of the microbes and significantly escalate the diversity. It was also supported by Zamorska and Papciak [24] explaining that diatomite capable of arousing the growth of microbes which quicken the development of biofilm. It acts as a buffer medium and nutrients and makes the microbes overgrow quickly on the diatomite surface. 
Table 1

Alpha Diversity of Seed Sludge, AGS Control and AGS Diatomite

\begin{tabular}{|lllll|}
\hline Alpha diversity & Sequence & Chao & Shannon & Simpson \\
\hline Seed Sludge & 392980 & 222 & 5.609 & 0.95 \\
\hline AGS Control & 335020 & 217 & 6.005 & 0.97 \\
\hline AGS Diatomite & 317440 & 207 & 6.013 & 0.97 \\
\hline
\end{tabular}

\subsection{The Microbial Community of Seed Sludge, Control AGS and AGS Diatomite}

The performance and development of aerobic granules were highly influenced by the microbial community. In this study, 16 s ion metagenomics analysis was performed to analyze the microbial diversity in seed sludge, AGS control and AGS diatomite samples. Table 2 shows the relative abundance percentage of each microbial domain in all three samples. The microbial domain discovered were bacteria, eukaryote, archaea and virus. From Table 2, the results for microbial domain's relative abundance in each sample were different. Interestingly, AGS diatomite has more diversity compared to seed sludge and controlled AGS. It was noticed that $99.36 \%$ of bacteria in seed sludge were reduced to $98.36 \%$. While, AGS control only experienced a minor change in the relative abundance of bacteria, $99.2 \%$. A reduction in bacteria abundance indicates that only functional bacteria that assist in granulation process and removal performance were left in the system. Another apparent change was the increase of eukaryote from $0.62 \%$ in seed sludge to $0.75 \%$ AGS control and $1.59 \%$ in AGS diatomite. Moreover, archaea and virus were in small abundance for all samples with the highest $0.05 \%$ in AGS diatomite. The change in domain-relative abundance signifies the evolution of microbial communities from seed sludge sample to aerobic granules, AGS control and AGS diatomite. A lot of researchers mentioned the shift of microbial community during granulation was due to the adjustment of the operational parameter in bioreactor system such as settling time, organic loading rate and aggregation characteristic [21, 25, 27]. For instance, the type of microbes that operated with short settling time, would retain fast settling microbes and eliminated slow settling microbes. In other words, the microbes that remained throughout granulation would have a beneficial function which influences the ability and characteristic of AGS in the system.

Table 2

Domain distribution of Seed Sludge, AGS Control and AGS Diatomite

\begin{tabular}{|llll|}
\hline Domain & Seed Sludge & AGS control & AGS diatomite \\
\hline Bacteria & $99.36 \%$ & $99.2 \%$ & $98.36 \%$ \\
\hline Eukaryote & $0.62 \%$ & $0.75 \%$ & $1.59 \%$ \\
\hline Archaea and Virus & $0.02 \%$ & $0.03 \%$ & $0.05 \%$ \\
\hline
\end{tabular}


Specifically, the microbial community comprised of bacteria, eukaryote, archaea and virus with different function and behavior. Starting with bacteria, the largest domain in the microbial community. Within the bacteria domain, there are many functional bacteria discovered such as filamentous bacteria, denitrifying bacteria, nitrifying bacteria and phosphorus accumulating bacteria. According to Rosman [27], bacteria possess the most versatile characteristic among all organism as they have unique nutrients necessity, as well as catabolic and metabolic activities. Furthermore, bacteria played crucial roles in wastewater treatment and the granulation process. They can degrade pollutants and a majority of them could secrete EPS for the AGS formation and stabilization. All the sample's microbial community were predominantly filled with bacteria domain with more than $98 \%$ relative abundance compared to other domains. Next, the second domain in the microbial community was eukaryote. Eukaryote is an organism that consumed bacterial cells and detritus. Eukaryotic organisms normally engage in aerobic granulation process as it could lead to the formation of filamentous structure which initiates the formation of the granules. Moreover, they also could act as the backbone to strengthen the structure especially for small size granules [28]. Chan et al. [29] stated that Sessile ciliates (protozoa) were discovered in high abundance on the surface of granules. These ciliates could feed on suspended bacteria and also act as a form of substratum for bacteria colonization. In contrast, Chan et al. [29], also mentioned the small population of eukaryote in the microbial community suggest that it does not have a significant impact towards the formation of aerobic granules. Yet, eukaryote could provide selective pressure for granules development even though did not directly involve in granular structure [21]. Based on the analysis, AGS diatomite's microbial community contained the highest eukaryote relative abundance compared to other samples.

Archaea and virus domain takes up the smallest abundance percentage in the microbial community. In aerobic granules, being minor domains, an archaeal organism might not influence the degradation activity for both nutrients and organic matter. However, previous studies explained that archaea have some minor roles that affected the properties of the granules [30]. As reported by Zhang et al. (2020), archaea were an important element for ensuring the stability and dense structure of AGS as they act as a nucleus for the granulation to take place. Gonzalez-Martinez et al. [31] also explained that the archaea community could build metabolic pathways in the aerobic granules that would enhance the performance of the treatment. Nonetheless, different case occurred to virus domain. The virus was considered harmful to the environment. Therefore, it was important to monitor and remove the virus in the wastewater and prevent further spread after the treatment.

\subsection{Comparison of Bacterial Community between Seed sludge, AGS control and AGS diatomite samples}

Bacteria were the most dominant domain in all three samples. The large percentage of bacteria in the microbial community makes them significantly important for microbial analysis. The bacteria diversity in all samples were a contrast in all taxonomy levels. The major population of bacteria in seed sludge normally appeared differently from those in granular samples. AGS diatomite with the presence of diatomite is seen as a new character to be compared with seed sludge and granular samples. In previous alpha diversity discussions, diatomite recorded the highest diversity among all sample. High diversity of 
bacterial community was one of the factors influencing the formation of aerobic granules [32]. Thus, in this section, the identification of relative abundance for bacteria diversity in the seed sludge, AGS control and AGS diatomite are compared. Figure 2 and Figure 3 illustrated the bacteria relative abundance at the phylum and class levels for all sample, respectively. The difference in the relative abundance of bacteria diversity between the three samples was spotted in each level.

\section{(1) Phylum Levels}

Referring to Figure 2, the bacteria population in seed sludge was different from AGS control and AGS diatomite. The seed sludge sample consisted of 11 phyla while AGS control contained 10 phyla and AGS diatomite has a minimum of 8 phyla respectively. It proved the microbial richness of seed sludge having the most number of phyla compared to control and AGS diatomite. Nonetheless, even though the number of phyla in AGS diatomite was minimum, each of them comprised high relative abundance. This signifies the high diversity of AGS diatomite sample in their bacterial community. According to Harun [21], throughout the granulation process, a specific type of bacteria was preserved in the system. They undergo the adaption process and presumably being part of the elements that contribute to the granulation process. The phylum that preserved after the granulation in AGS control were Proteobacteria, Bacteroidetes, Actinobacteria, Nitrospirae, Firmicutes, Gemmatimonadetes Acidobacteria, Verrucomicrobia, Chloroflexi and Ignavibacteriae. Correspondingly, all of the phylum in AGS control were preserved in AGS diatomite except Chloroflexi and Ignavibacteriae.

In phylum levels, the most abundant bacteria that comprised more than $10 \%$ of the bacteria population were Proteobacteria, Bacteroidetes, Actinobacteria, and Nitrospirae. Among all, Proteobacteria had the highest percentage abundance in all three samples. The seed sludge sample recorded a percentage of $63 \%$ which was the highest abundance compared to $38 \%$ in control and $46 \%$ in AGS diatomite respectively. According to Yan et al. [33], Proteobacteria are the common leading phylum in all kind of samples and a crucial element for the granulation process. A similar finding was discovered by Guo et al. [4] and $\mathrm{He}$ et al. [7] as the Proteobacteria was the most dominant in their respective bacterial community. Othman [32] explained, the dominancy of Proteobacteria might suggest that it have a significant contribution to the granulation process. Proteobacteria have the capability to increases the production of EPS which promotes the adhesion of floc sludge to become granules. Besides, it also enhances the granules' ability on the removal performance of $\mathrm{COD}$ and $\mathrm{NH}_{3}-\mathrm{N}$ [34]. Surprisingly, it was observed that AGS diatomite was slightly better than controlled AGS in the abundance percentage of Proteobacteria. This presumably related to the presence of diatomite which has the ability to attract functional bacteria to grow on its surface.

Meanwhile, the second dominant phylum was Phylum Bacteroidetes. It was a well-known primary bacterium in most of the aerobic granules developed in municipal and domestic wastewater. Phylum Bacteroidetes was abundance in controlled AGS with the percentage of $26 \%$ compared to $20 \%$ in AGS diatomite and $13 \%$ in seed sludge. Bacteroidetes was mainly responsible for nitrification process of the AGS. It helps in regulating the autotrophic metabolism and nitrite-oxidizing during the treatment process 
[34]. Dahalan [35] mentioned Bacteroidetes normally discovered in the inner core of the AGS where there was a limited amount of oxygen. Bacteroidetes also could enhance EPS production which increases the cell hydrophobicity of the granules and promote the granulation process [32]. Latterly, various studies discovered Bacteroidetes as a dominant bacterial population found in AGS [22,36,37]. As the sludge going through a granular transformation, the abundance percentage of this phylum will keep on increasing along with the process. It could be observed by the smaller percentage of Bacteroidetes in seed sludge as compared to granules (AGS control and AGS diatomite). However, excessive Bacteroidetes phylum could damage the stability of the structure of the granules [38].

Next, Actinobacteria phylum were abundance as well with a percentage of $13 \%$ in controlled AGS and $9 \%$ equal percentages for both seed sludge and AGS diatomite. Dahalan [35] stated high abundance of Actinobacteria were beneficial for the formation of AGS. It was reported by Song et al. [39] that most of the bacteria extracted from aerobic granules belonged to Actinobacteria class. Wang et al. [34] also stated that Actinobacteria responsible in nitrification and denitrification process. In this study, both AGS (AGS control and AGS diatomite) have a high abundance of Actinobacteria which possibly related to the efficient removal of ammonia nitrogen achieved during the experiment. Another abundant phylum was Nitrospirae. It was noticed that the seed sludge, controlled AGS and AGS diatomite have approximately similar percentage abundance with a proportion of $11 \%, 11 \%$ and $10 \%$ respectively. Nitrospirae was responsible for complete oxidation of ammonia to nitrite and nitrate in the system. Morales et al. [40] explained Nitrospirae phylum was also known as Nitrite oxidizing bacteria (NOB) and normally dominant in the majority of the wastewater treatment plant. The similar relative abundance for all the samples implies the diatomite do not influence in promoting Nitrospirae bacteria.

Conspicuously, phylum Gemmatimonadetes was higher in AGS diatomite than other samples. AGS diatomite recorded $7 \%$ percentage abundance whilst controlled AGS and seed sludge only attained $1 \%$ and $0.1 \%$ individually. It could be assumed that Gemmatimonadetes bacteria were clearly attracted towards diatomite and eventually became an important component of the granules. Gemmatimonadetes was known as polyphosphate accumulating microorganism that in charge of the removal of phosphate from wastewater [41]. This may act as one of the factors that boost the AGS diatomite performance for $\mathrm{PO}_{4}-\mathrm{P}$ removal after only 50 days of the experiment. Other phyla such as Firmicutes and Acidobacteria were also found with high abundance in granules than seed sludge. This result was comparable to Liu et al. [42]. Firmicutes were classified as heterotrophs while Acidobacteria were autotrophs. Both phyla can consume carbon and nitrite compound as well as producing EPS for microbial granulation. Subsequently, Chloroflexi, Ignavibacteriae, Sphirochaetes and Verrucomicrobia were also included and considered minor phylum as most of them were present in seed sludge comprising low percentage abundance.

(2) Class Levels

In class levels, four major subclasses of Phylum Proteobacteria involving a-Proteobacteria, $\beta$ Proteobacteria, $\gamma$-Proteobacteria and $\delta$-Proteobacteria were discovered for all three samples. As demonstrated in Figure 3 , among all subclasses, $\beta$-Proteobacteria attained the biggest fraction of 
Proteobacteria accounting for $38 \%$ in seed sludge, $16 \%$ in AGS control and $24 \%$ in AGS diatomite. It was followed by $\mathrm{Y}$-Proteobacteria obtaining $7 \%, 13 \%$ and $12 \%$ for all the samples respectively. aProteobacteria was the approximately similar in all samples acquiring $8 \%$ seed sludge, $7 \%$ AGS control and $7 \%$ AGS diatomite. Also, $\delta$-Proteobacteria achieving the highest percentage abundance in seed sludge (9\%) as compared to AGS control (3\%) and AGS diatomite (2\%). Notably, all of these classes under Proteobacteria have their roles inside the granules. The most dominant $\beta$-Proteobacteria were a crucial component commonly present in AGS. Harun [21], explained that $\beta$-Proteobacteria were responsible for compactness and strength of the structure of the granules. $\beta$-Proteobacteria were the most popular aerobic nitrogen bacteria that involves nitrifying and denitrifying bacteria. It was supported by Wang et al. [37] claiming $\beta$ - Proteobacteria has the ability of degradation which play a critical role towards the removal of ammonia nitrogen and biological nitrogen.

Subsequently, $Y$-Proteobacteria was one of the classes that significantly improved after granules development. $Y$-Proteobacteria could cultivate aerobically in low substrate condition which was the case for this study. It could store carbon source as intracellular polyhydroxyalkanoates (PHA) as well as undergo biodegradation of nitrogen compounds. Exceptionally, they were an essential element of AGS that is well known for producing glue-like EPS for cells bondings. Other than that, $y$-Proteobacteria also involves in denitrification and phosphorus removal and able to endure harsh condition [7]. It was also mentioned by $\mathrm{He}$ et al. [7] that $\beta$-Proteobacteria, 囚- Proteobacteria, and could resist shear force and contribute considerably on the formation of AGS. Next, a-Proteobacteria that also known as filamentous bacteria were often associated with nitrification process and the initial stage of granules formation. This type of filamentous bacteria could be attached to other microbes and among themselves forming a backbone that leads to the development of the aggregate. a-Proteobacteria appeared to be robust and large morphology and regularly dominant in industrial wastewater treatment system. The same amount of relative abundance of a-Proteobacteria in all samples were comparable with Wang et al. [37] which suggest it was preserved and became part of the granules. Whereas for $\delta$-proteobacteria, were observed to experience a clear reduction in relative abundance from seed sludge to granules during the succession of microbial community and development of AGS. Wu et al. [13] discovered the same circumstances and explained, $\delta$-proteobacteria does not have a big influence for granules formation. Nonetheless, Zhang et al. [43] highlighted that $\delta$-proteobacteria consist of NOB which responsible in converting the nitrite to nitrate. Thus, it could enhance the nitrite removal in the treatment system.

Afterwards, in the second abundance phylum of Bacteroidetes, there are 2 major classes were observed in all samples. There are Sphingobacteriia and Cytophagia accounted for $15 \%$ in controlled AGS, $11 \%$ in seed sludge and $9 \%$ in AGS diatomite. Similar findings also discovered by Luo et al. [44] as Sphingobacteriia were dominant in both seed sludge and granules. Until now, there was no clear indication of Sphingobacteriia influence towards the formation of the granules. However, Świątczak and Cydzik-Kwiatkowska [45] explained that Sphingobacteriia were heavily involved in the denitrification process. Saprospiraceae, family level of Sphingobacteriia were recognized as autotrophic denitrifiers and can degrade protein and hydrocarbon. Meanwhile, another dominant class were Cytophagia. AGS diatomite obtained the most relative abundance with $8 \%$ followed by controlled AGS, $6 \%$ and seed sludge, 
$1 \%$. The abundance of Cytophagia in both granules indicates its participation during the granulation process. Furthermore, the result also suggested the Cytophagia possibly one of bacteria class attracted to the diatomite. According to Świątczak and Cydzik-Kwiatkowska [45], Cytophagia were classified as facultative bacteria that mainly responsible for organic matter removal. It also highly involved in the granulation process as a structural microorganism [37]. In addition, Cytophagia class played an integral role in ensuring stable operation by removing the pollutants.

Other dominant classes such as Nitrospira and Actinobacteria were similar in abundance to their phylum. Both of them were the only class detected and no other classes appeared in each Nitrospirae and Actinobacteria phylum. The percentage abundance of Nitrospira class in seed sludge, AGS control and AGS diatomite were approximately comparable to each other with $11 \%, 11 \%$ and $10 \%$ respectively. The same case occurred to Actinobacteria accounting for $9 \%$ in both seed sludge and AGS diatomite sample as well as $13 \%$ in controlled AGS. Both classes possessed the same capability with their parent phylum involving the enhancement of nitrification and denitrification process. In the case of AGS diatomite, Gemmotimonadetes were another single class observed in the community acquiring $7 \%$ percentage abundance. Lastly, the sudden emergence of bacili and clostridia class under Firmicutes phylum were noticed in AGS diatomite and AGS control. In seed sludge, the relative abundance of these bacteria classes was very low. Bacili was a popular aerobic bacteria and mostly present in AGS. According to Guo et al. [4], Bacili microbes plays a skeletal role in aerobic granules formation. It contributes to the microbial morphology and normally attached with cocci microorganism. Bacili is also capable to produce shortchain acids from many substrates, such as glucose, fructose, mannose, or starch for growth of the granules [46]. Meanwhile, He et al. [7] stated that Clostridia was highly associated with nitrification and denitrification process. It also has the ability to accumulate polyhydroxy butyrate (PHB) into the cells which enable simultaneous removals of phosphorus and nitrogen from the system.

\section{(3) Bacteria Involves in Granulation}

The mechanism of granules formation is closely related to the interaction of microbial entities and the surrounding condition. The microbes prefer a dispersed rather than aggregated state [47]. Thus, it is crucial to have an initiating force that could make them joint with each other and finally, form an aggregate. One of the key elements that contribute to the adhesion phenomenon and matrix structure formation in AGS is the extracellular polymeric substance (EPS). EPS is secreted by microbes and function as a bio glue which facilitates the cell-to-cell adhesion of microbial biomass during the granulation process [5]. According to Sheng et al. [48], EPS have a huge influence on the physicochemical properties of AGS. EPS is made up of exopolysaccharides, exoproteins, DNA, humic acid and uronic acid which affect the properties of the granules including structure stability, surface charge, hydrophobicity and adsorption ability. Notably, a high population of EPS producing microbes could significantly increase EPS production, which leads to a stable and dense structure of granules. It was supported by Xia et al. [49], explaining that the instability of AGS was due to the decrease of EPS producers in the granules. Therefore, the microbes that potentially produced EPS was identified in this study by comparing their relative abundance in seed sludge, AGS control and AGS diatomite. 
The top 21 EPS producing bacteria was identified in all three samples and listed in Table 3. Overall, the sample with the highest total abundance of EPS bacteria was AGS diatomite attaining $68 \%$ while AGS control and seed sludge recorded $51 \%$ and $29.3 \%$ respectively. Based on the result, seed sludge has the lowest abundance of EPS bacteria compare to the granules. It was supported by Zhang et al. [50] explaining that the granulation process promotes the enrichment of EPS bacteria to increase the production of "bio glue" for cells adhesion. Xia et al. [49] also reported the EPS content was higher in aerobic granules than seed sludge. Meanwhile, in the case of granules, there was a huge difference in the percentage abundance of EPS bacteria between AGS diatomite and AGS control. A difference of $17 \%$ percentage abundance was observed between both granules. This suggests that there was a high possibility of diatomite capability to attract EPS producing bacteria towards its surface. Besides, a higher percentage of EPS producing bacteria in AGS diatomite indicates high EPS content that supported the granulation process compare to AGS control. Specifically, the dominant EPS producing bacteria observed in AGS diatomite were Cytophagales, Xanthomonadales, Rhodocyclales, Comamonadaceae, Gemmatimonadales and Chitinophagaceae. 
Table 3

EPS Producing Bacteria in Seed Sludge, AGS Control and AGS Diatomite

\begin{tabular}{|c|c|c|c|c|c|}
\hline No. & EPS Microbial & $\begin{array}{l}\text { Seed } \\
\text { Sludge }\end{array}$ & $\begin{array}{l}\text { AGS } \\
\text { control }\end{array}$ & $\begin{array}{l}\text { AGS } \\
\text { diatomite }\end{array}$ & References \\
\hline 1 & Xanthomonadales & $3 \%$ & $10 \%$ & $10 \%$ & $\begin{array}{l}\text { Shahnavaz et al. } \\
\text { (2015) }\end{array}$ \\
\hline 2 & Cytophagales & $1 \%$ & $6 \%$ & $8 \%$ & $\begin{array}{l}\text { Rusanowska et al. } \\
\text { (2019) }\end{array}$ \\
\hline 3 & Rhodocyclales & $8 \%$ & $7 \%$ & $10 \%$ & Xia et al. (2018) \\
\hline 5 & Comamonadaceae & $6 \%$ & $4 \%$ & $7 \%$ & Xia et al. (2018) \\
\hline 6 & Gemmatimonadales & $0.4 \%$ & $1 \%$ & $7 \%$ & Fawaz (2013) \\
\hline 7 & Chitinophagaceae & $1 \%$ & $6 \%$ & $6 \%$ & NGUON (2017) \\
\hline 8 & Clostridiales & $2 \%$ & $4 \%$ & $3 \%$ & Mckew et al. (2013) \\
\hline 9 & Bacteroidales & $0.9 \%$ & $4 \%$ & $3 \%$ & McKew et al. (2013) \\
\hline 10 & Chromatiales & $2 \%$ & $3 \%$ & $2 \%$ & Cruz (2014) \\
\hline 11 & Verrucomicrobiae & $0.1 \%$ & $0.3 \%$ & $2 \%$ & $\begin{array}{l}\text { Rusanowska et al. } \\
\text { (2019) }\end{array}$ \\
\hline 12 & Bacilli & $1 \%$ & $3 \%$ & $2 \%$ & $\begin{array}{l}\text { Irnov and Winkler } \\
(2010) .\end{array}$ \\
\hline 13 & Rhizobiales & $2 \%$ & $1 \%$ & $3 \%$ & $\begin{array}{l}\text { Mukherjee et al. } \\
(2011)\end{array}$ \\
\hline 14 & Flavobacteriia & $0.2 \%$ & $0.2 \% \%$ & $2 \%$ & $\begin{array}{l}\text { Świątczak et al. } \\
(2019)\end{array}$ \\
\hline 15 & Thauera & $0.8 \%$ & $0.9 \%$ & $1 \%$ & $\begin{array}{l}\text { Rusanowska et al. } \\
\text { (2019) }\end{array}$ \\
\hline 16 & Paracoccus & $0.1 \%$ & $0.03 \%$ & $0.2 \%$ & $\begin{array}{l}\text { Świątczak et al. } \\
(2019)\end{array}$ \\
\hline 17 & Staphylococcaceae & $0 \%$ & $0.3 \%$ & $0.7 \%$ & Costa et al. (2018) \\
\hline 18 & Pseudomonadales & $0.4 \%$ & $0.1 \%$ & $0.6 \%$ & $\begin{array}{l}\text { Rusanowska et al. } \\
\text { (2019) }\end{array}$ \\
\hline 19 & Methylococcales & $0.06 \%$ & $0.04 \%$ & $0.2 \%$ & Harik (2016) \\
\hline 20 & Acidovorax & $0 \%$ & $0.08 \%$ & $0.03 \%$ & Xia et al. (2018) \\
\hline 21 & Zoogloea & $0.7 \%$ & $0.09 \%$ & $0.3 \%$ & Xia et al. (2018) \\
\hline 22 & Acidobacteria & $0.5 \%$ & $0.2 \%$ & $2 \%$ & Liu et al. (2017) \\
\hline
\end{tabular}




\begin{tabular}{|lllll|}
\hline No. EPS Microbial & $\begin{array}{l}\text { Seed } \\
\text { Sludge }\end{array}$ & $\begin{array}{l}\text { AGS } \\
\text { control }\end{array}$ & $\begin{array}{l}\text { AGS } \\
\text { diatomite }\end{array}$ & References \\
\hline $\begin{array}{l}\text { Total percentage abundance of EPS } \\
\text { bacteria }\end{array}$ & $29 \%$ & $51 \%$ & $68 \%$ & \\
\hline
\end{tabular}

Among the listed bacteria, it was noticed that Xanthomonadales experienced the most significant increase in relative abundance after granulation for both AGS. Its' relative abundance evolved from only $3 \%$ in seed sludge to $10 \%$ in both AGS indicating the crucial role played by this bacterium for the structure of the granules. According to Zhang et al. [50], Xanthomonadales were frequently detected in AGS and normally enriched in young granules. Moreover, this type of bacteria also categorized as denitrifiers which highly involved in nitrification and denitrification process. Xia et al. [49] and Shahnavaz et al. [51] explained Xanthomonadales was considered as a crucial character that associated with EPS production and greatly responsible for the granulation process. Notably, Xanthomonadales belong to class Gammaproteobacteria that are well-known EPS producer.

Subsequently, Cytophagales which belongs to phylum Bacteroidetes was another bacterium that has an obvious rise in relative abundance after granulation. Before undergoing granulation, its relative abundance in seed sludge was $1 \%$. While, after granulation, it achieved $6 \%$ and $8 \%$ for controlled AGS and AGS diatomite accordingly. It was backed by Świątczak et al. [52] stating that Cytophagia (class of Cytophagales) abundance was greater in mature aerobic granules. This circumstance gave an indication of the maturity of AGS diatomite having the highest abundance of Cytophagales as compared with controlled AGS. Cytophagales could produce EPS and also involve in granulation process as structural microbes [53]. Additionally, Cyctophagales largely responsible for the removal of organic and nutrients from the treatment system. The abundance of Cytophagales normally increased by three times after granulation and significantly improved the treatment performance [52].

Different from Xanthomonadales and Cytophagales that high in abundance in granules, Gemmatimonadales was a special case. Gemmatimonadales was extremely low abundance in seed sludge with only $0.4 \%$ while AGS control also recorded $1 \%$. Surprisingly, it was observed that the relative abundance of Gemmatimonadales in AGS diatomite was 7\%. The huge difference between controlled AGS and AGS diatomite suggest the presence of diatomite presumably favour the growth of Gemmatimonadales. According to Shchytov [41], Gemmatimonadales belongs to phylum Gemmatimonadetes and was identified as a polyphosphate accumulating microbes as well as PHA accumulating microbes in the wastewater treatment system. It also has a phenomenal capability that makes it able to survive in both aerobic and anaerobic condition [42]. Moreover, a detailed study of phylum Gemmatimonadetes conducted by Fawaz [54] discovered the ability of this bacteria to produce EPS. Thus, Gemmatimonadales was an important EPS producing bacteria in AGS diatomite which undoubtedly contributes to the granulation process. Other than that, Gemmatimonadales also involves in phosphate removal which might be the case for exceptional phosphate removal performance of AGS diatomite compared to AGS control. 
Next, Chitinophagaceae bacteria which belongs to phylum Bacteroidetes was also an EPS producer. The relative abundance was high in AGS control and AGS diatomite with $6 \%$ as compared to $1 \%$ in seed sludge. This bacterium seems to commonly increase in abundance after the granulation process to enhance the structural stability of granules. Meanwhile, other dominant EPS producing bacteria including Rhodocyclales and Comamonadaceae were discovered to have approximately similar abundance in all samples. Given there was no significant change in abundance after granulation, it was uncommon circumstances as Xia et al. [49] explained that these bacteria were hugely responsible for the granulation process. This suggests that Rhodocyclales and Comamonadaceae abundance were possibly high during the granulation process and after the granules attained maturity, they slowly disappear. Therefore, all the EPS producing bacteria listed in Table 4.6 including the minor genera have their level of contribution towards the formation and stability of the granules.

\section{Conclusions}

The formation of AGS was enhanced by diatomite compare to the natural granulation process. AGS diatomite achieve granulation after 21 days while control AGS 85 days. Afterward, the metagenomics analysis has discovered a different relative abundance of the microbial community in the seed sludge, AGS control (90 days) and AGS diatomite (30 days) samples. The findings revealed AGS diatomite have a higher microbial diversity than AGS control and seed sludge. Diatomite can promote growth and increase the relative abundance of functional bacteria. It was proved after AGS diatomite recorded the highest Shannon index, 6.013 for alpha diversity while AGS control attained 6.005. Moreover, the EPS producing bacteria were significantly increased in abundance in AGS diatomite compared to AGS control. There were 68\% EPS bacteria discovered in AGS diatomite higher than AGS control, 51\% from the seed sludge (29\%). The most dominant bacteria in AGS diatomite which enhanced after granulation were Xanthomonadales, Cytophagales and Gemmatimonadales. Notably, the most interesting discovery about the addition of diatomite was the high abundance of phylum Gemmatimonadetes. Phylum Gemmatimonadales was highly attracted to diatomite compared to other samples. With the ability to produce EPS, removed phosphate and accumulates PHA, Gemmatimonadales presumably the key player in the AGS diatomite microbial community.

\section{Declarations}

\section{Availability of data and materials}

All data generated or analyzed during this study are available to view on all articles.

\section{Competing interests}

The authors declare they have no competing interests.

\section{Funding}


This work was supported by prototype development research grant scheme (Grant No: R.K 130000.7843.4L682).

\section{Authors' contributions}

Corresponding author, Hazlami Fikri Basri provided the data analysis and manuscript. All authors read and approved the final manuscript.

\section{Acknowledgements}

The authors would like to also express gratitude to the Malaysia-Japan International Institute of Technology for the scholarship granted to the authors.

\section{References}

1. Basri HF, Anuar AN, and Ab Halim MH. Pilot scale study on characterization and performance of aerobic granular sludge to treat domestic wastewater. Malaysian Journal of Fundamental and Applied Sciences. 2020;16:1: 38-43.

2. Bengtsson $S$, de Blois $M$, Wilén $B M$, and Gustavsson $D$. A comparison of aerobic granular sludge with conventional and compact biological treatment technologies. Environ. Technol. 2019;40:21:27692778.

3. Pronk M, Abbas B, Al-Zuhairy SHK, Kraan R, Kleerebezem R and Van Loosdrecht MCM. Effect and behaviour of different substrates in relation to the formation of aerobic granular sludge. Appl. Microbiol. Biotechnol. 2015;99:12: 5257-5268

4. Guo K, Shang Y, Gao B, Xu X, Lu S, and Qi Q. Study on the treatment of soybean protein wastewater by a pilot-scale IC-A/O coupling reactor. Chem. Eng. J. 2018;343:189-197.

5. Winkler MKH, Meunier C, Henriet O, Mahillon J, Suárez-Ojeda ME, Del Moro G, De Sanctis M, Di laconi $\mathrm{C}$ and Weissbrodt DG. An integrative review of granular sludge for the biological removal of nutrients and recalcitrant organic matter from wastewater. Chem. Eng. J. 2018;336:489-502.

6. Lv Y, Wan C, Lee D J, Liu X, and Tay JH. Microbial communities of aerobic granules granulation mechanisms. Bioresour. Technol. 2014;169:344-351.

7. He Q, Chen L, Zhang S, Chen R, Wang H, Zhang W and Song J. Natural sunlight induced rapid formation of water-born algal-bacterial granules in an aerobic bacterial granular photo-sequencing batch reactor. J. Hazard. Mater. 2018;359:222-230.

8. Chen C, Ming J, Yoza BA, Liang J, Li QX, Guo H, Liu Z, Deng J and Wang Q. Characterization of aerobic granular sludge used for the treatment of petroleum wastewater. Bioresour. Technol. 2019;271:353-359.

9. De Sousa Rollemberg SL, Barros ARM, de Lima JPM, Santos AF, Firmino PIM, and dos Santos AB. Influence of sequencing batch reactor configuration on aerobic granules growth: Engineering and microbiological aspects. J. Clean. Prod. 2019;238:117906. 
10. Jiang Y, Wei L, Yang K, and Wang H. Investigation of rapid granulation in SBRs treating aniline-rich wastewater with different aniline loading rates. Sci. Total Environ. 2019;646,841-849.

11. Liang Z, Tu Q, Su X, Yang X, Chen J, Chen Y, Liu C, Li H and He Q. Formation, extracellular polymeric substances and microbial community of aerobic granules enhanced by microbial flocculant compared with poly-aluminum chloride. J. Clean. Prod. 2019;220:544-552.

12. Liu X, Chen Y, Zhang X, Jiang X, Wu S, Shen J, Sun X, Li J, Lu L and Wang L. Aerobic granulation strategy for bioaugmentation of a sequencing batch reactor (SBR) treating high strength pyridine wastewater. J. Hazard. Mater. 2015;295:153-160.

13. Wu D, Zhang Z, Yu Z, and Zhu L. Optimization of F/M ratio for stability of aerobic granular process via quantitative sludge discharge. Bioresour. Technol. 2018;252:150-156.

14. Hamza RA, lorhemen OT, Zaghloul MS, and Tay JH. Rapid formation and characterization of aerobic granules in pilot-scale sequential batch reactor for high-strength organic wastewater treatment. J. Water Process. Eng. 2018;22:27-33.

15. Gu QY, Liu XY and Wang ZN. Enhanced Performance of Sulfate Reducing Bacteria (SRB) Granules Process by Using Diatomite as Functional Carrier Material. Key Eng. Mater. 2017;730:206-211.

16. Xu K, Liu Y, Wang Y, Wang T, Wang H, Liang X, Lu C, Tan Y, Liu X and Wang P. A Novel Wastewater Treating Material: Cationic Poly Acrylamide/Diatomite Composite Flocculants. J Polym Environ. 2018;26:7:305-3059.

17. Sguanci S, Lubello C, Caffaz S, and Lotti T. Long-term stability of aerobic granular sludge for the treatment of very low-strength real domestic wastewater. J. Clean. Prod. 2019;222:882-890.

18. Sarma SJ, Tay JH and Chu A. Finding knowledge gaps in aerobic granulation technology. Trends Biotechnol. 2017;35:1:66-78.

19. Kamaruddin NF. Diatomite as Natural Coagulant in the Removal of Water Turbidity. Master of Philosophy, Universiti Teknologi Malaysia, Kuala Lumpur; 2017.

20. Chen C, Liang J, Yoza BA, Li QX, Zhan Y, and Wang Q. Evaluation of an up-flow anaerobic sludge bed (UASB) reactor containing diatomite and maifanite for the improved treatment of petroleum wastewater. Bioresour. Technol. 2017;243:620-627.

21. Harun, H. Treatment System for Soy Sauce Wastewater through Granular Sequencing Batch Reactor. Doctor of Philosophy, Universiti Teknologi Malaysia, Skudai; 2016.

22. Zou J, Pan J, Wu S, Qian M, He Z, Wang B, and Li J. Rapid control of activated sludge bulking and simultaneous acceleration of aerobic granulation by adding intact aerobic granular sludge. Sci. Total Environ.2019;674:105-113.

23. Yang GF, Feng LJ, Wang SF, Yang Q, Xu XY, and Zhu L. Performance and enhanced mechanism of a novel bio-diatomite biofilm pretreatment process treating polluted raw water. Bioresour. Technol. 2015;191:271-280.

24. Zamorska J, and Papciak D. Activity of nitrifying biofilm in the process of water treatment in diatomite bed. Environ. Prot. Eng. 2008;34:1:37. 
25. Yadav TC, Khardenavis AA and Kapley A. Shifts in Microbial Community in Response to Dissolved Oxygen Levels in Activated Sludge. Bioresour. Technol. 2014;165:257-264.

26. Rosman, N. Biogranular Sludge for Rubber Processing Wastewater in a Sequencing Batch Reactor. Doctor of Philosophy, Universiti Teknologi Malaysia, Skudai; 2017.

27. Sharaf A, Guo B, and Liu Y. Impact of the filamentous fungi overgrowth on the aerobic granular sludge process. Bioresour. Technol. Rep. 2019;7:100272.

28. Chan SH. Predator-prey interactions in aerobic granulation system. Doctor of Philosophy (IGS). Singapore: Nanyang Technological University, 2018;228

29. Muñoz-Palazon, B., Pesciaroli, C., Rodriguez-Sanchez, A., Gonzalez-Lopez, J., and Gonzalez-Martinez, A. (2018). Pollutants degradation performance and microbial community structure of aerobic granular sludge systems using inoculums adapted at mild and low temperature. Chemosphere, 204, 431-441.

30. Gonzalez-Martinez A, Muñoz-Palazon B, Maza-Márquez P, Rodriguez-Sanchez,A, Gonzalez-Lopez J, and Vahala R. Performance and microbial community structure of a polar Arctic Circle aerobic granular sludge system operating at low temperature. Bioresour. Technol. 2018;256:22-29.

31. Othman, I. Aerobic Granular Sludge from Different Cycle Time for Livestock Wastewater Treatment. Doctor of Philosophy, Universiti Teknologi Malaysia, Skudai; 2017.

32. Yan L, Zhang M, Liu Y, Liu, C, Zhang Y, Liu S, Yu L, Hao G, Chen Z and Zhang Y. Enhanced nitrogen removal in an aerobic granular sequencing batch reactor under low DO concentration: Role of extracellular polymeric substances and microbial community structure. Bioresour. Technol. 2019;289:121651.

33. Wang L, Zhan H, Wang Q, Wu G, and Cui D. Enhanced aerobic granulation by inoculating dewatered activated sludge under short settling time in a sequencing batch reactor. Bioresour. Technol. 2019;286:121386.

34. Dahalan, FA. Development and Characterization of Phototrophic Aerobic Granular Sludge. Doctor of Philosophy, Universiti Teknologi Malaysia, Skudai; 2012.

35. Liu J, Li J, Piché-Choquette S, and Sellamuthu B. Roles of bacterial and epistylis populations in aerobic granular SBRs treating domestic and synthetic wastewaters. Chem. Eng. J. 2018;351:952958.

36. Wang X, Chen Z, Shen J, Zhao X, and Kang J. Impact of carbon to nitrogen ratio on the performance of aerobic granular reactor and microbial population dynamics during aerobic sludge granulation. Bioresour. Technol. 2019;271, 258-265.

37. Tan G, Xu N, Liu Y, Hao H, and Sun W. Effects of lead concentration and accumulation on the performance and microbial community of aerobic granular sludge in sequencing batch reactors. Environ. Technol. 2016;3330:1-11.

38. Song Z, Pan Y, Zhang K, Ren N and Wang A. Effect of seed sludge on characteristics and microbial community of aerobic granular sludge. Journal of Environmental Sciences. 2010;22:9:1312-1318. 
39. Morales N, Figueroa M, Fra-Vázquez A, Del Río AV, Campos JL, Mosquera-Corral A, and Méndez R. Operation of an aerobic granular pilot scale SBR plant to treat swine slurry. Process Biochem. 2013;48:8:1216-1221.

40. Shchytov Oleksander. International Letters of Natural Sciences. 2019;74.

41. Liu L, Fan H, Liu Y, Liu C and Huang X. Development of algae-bacteria granular consortia in photosequencing batch reactor. Bioresour. Technol. 2017;232:64-71.

42. Zhang Y, Dong X, Liu S, Lei Z, Shimizu K, Zhang Z, Adachi Y. and Lee DJ. Rapid establishment and stable performance of a new algal-bacterial granule system from conventional bacterial aerobic granular sludge and preliminary analysis of mechanisms involved. J. Water Process. Eng. 2020;34:101073.

43. Luo J, Hao T, Wei L, Mackey HR, Lin Z, and Chen GH. Impact of influent COD/N ratio on disintegration of aerobic granular sludge. Water Res.2014;62:127-135.

44. Świątczak P, and Cydzik-Kwiatkowska A. Performance and microbial characteristics of biomass in a full-scale aerobic granular sludge wastewater treatment plant. Environ. Sci. Pollut. Res. 2018;25:2:1655-1669.

45. Lutpi NA, Jahim JM, Mumtaz T, Abdul PM, and Nor MTM. Physicochemical characteristics of attached biofilm on granular activated carbon for thermophilic biohydrogen production. RSC Adv. 2015;5:25:19382-19392.

46. Khan MZ, Mondal PK and Sabir S. Aerobic granulation for wastewater bioremediation: a review. Can. J. Chem. Eng. 2013;91:6:1045-1058.

47. Sheng GP, Yu HQ, and Li XY. Extracellular polymeric substances (EPS) of microbial aggregates in biological wastewater treatment systems: a review. Biotechnol. Adv. 2010;28:6:882-894.

48. Xia J, Ye L, Ren H, and Zhang XX. Microbial community structure and function in aerobic granular sludge. Applied microbiology and biotechnology, 2018;102:9:3967-3979.

49. Zhang Q, Hu J, and Lee DJ. Aerobic granular processes current research trends. Bioresour. Technol. 2016;210:74-80.

50. Shahnavaz B, Karrabi M, Maroof S, and Mashreghi M. Characterization and molecular identification of extracellular polymeric substance (EPS) producing bacteria from activated sludge. J. Cell. Mol. Med. 2015;7:2:86-93.

51. Świątczak P, Cydzik-Kwiatkowska A, and Zielińska M. Treatment of the liquid phase of digestate from a biogas plant for water reuse. Bioresour. Technol. 2019;276:226-235.

52. Rusanowska P, Cydzik-Kwiatkowska A, and Wojnowska-Baryła I. Microbial Origin of Excreted DNA in Particular Fractions of Extracellular Polymers (EPS) in Aerobic Granules. Water Air Soil Pollut. 2019;230:8:203.

53. Fawaz MN. Revealing the ecological role of gemmatimonadetes through cultivation and molecular analysis of agricultural soils. Master of Science, University of Tennessee, Knoxville; 2013. 


\section{Figures}
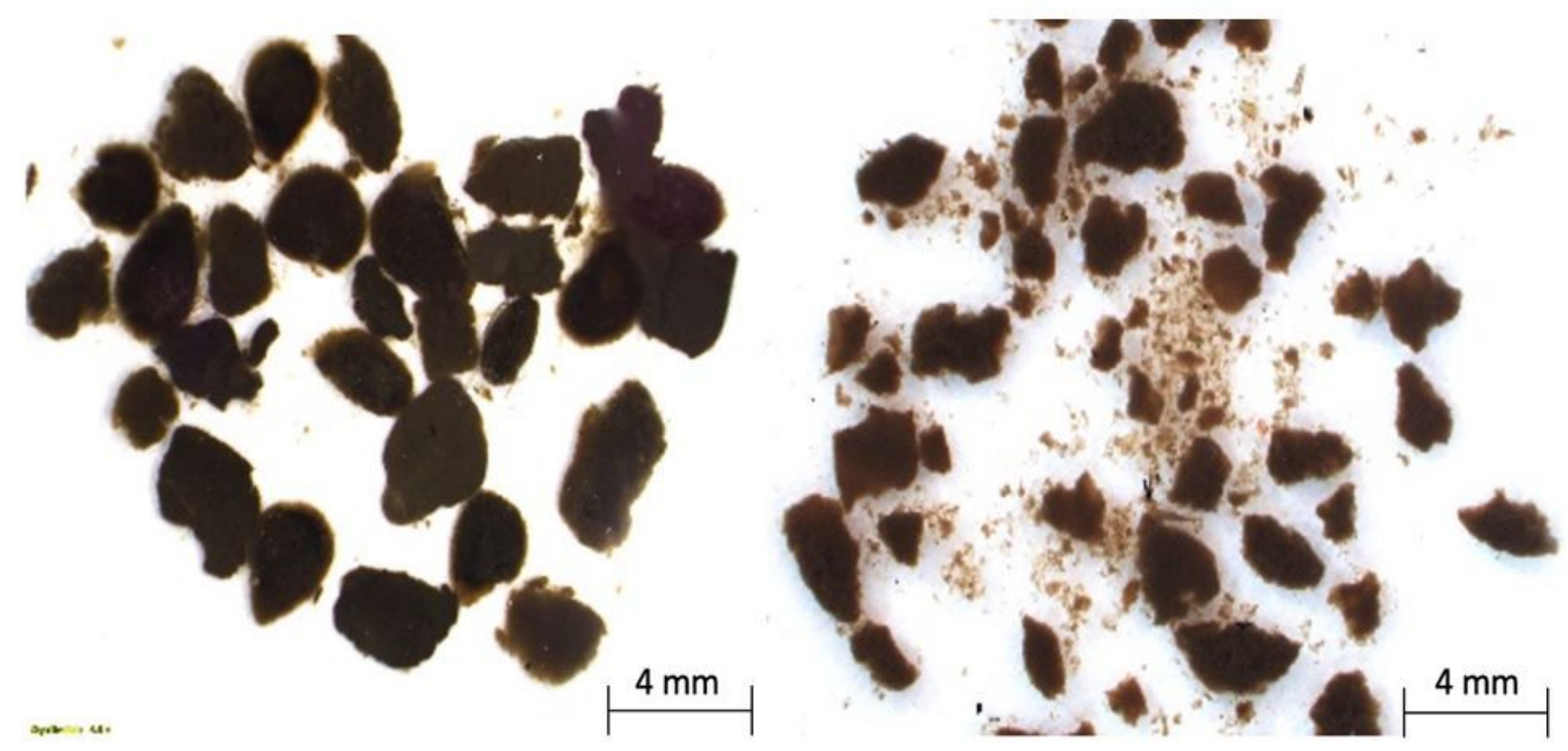

Figure 1

Microscopic images of a) AGS diatomite after 30 days and AGS control after 90 days 


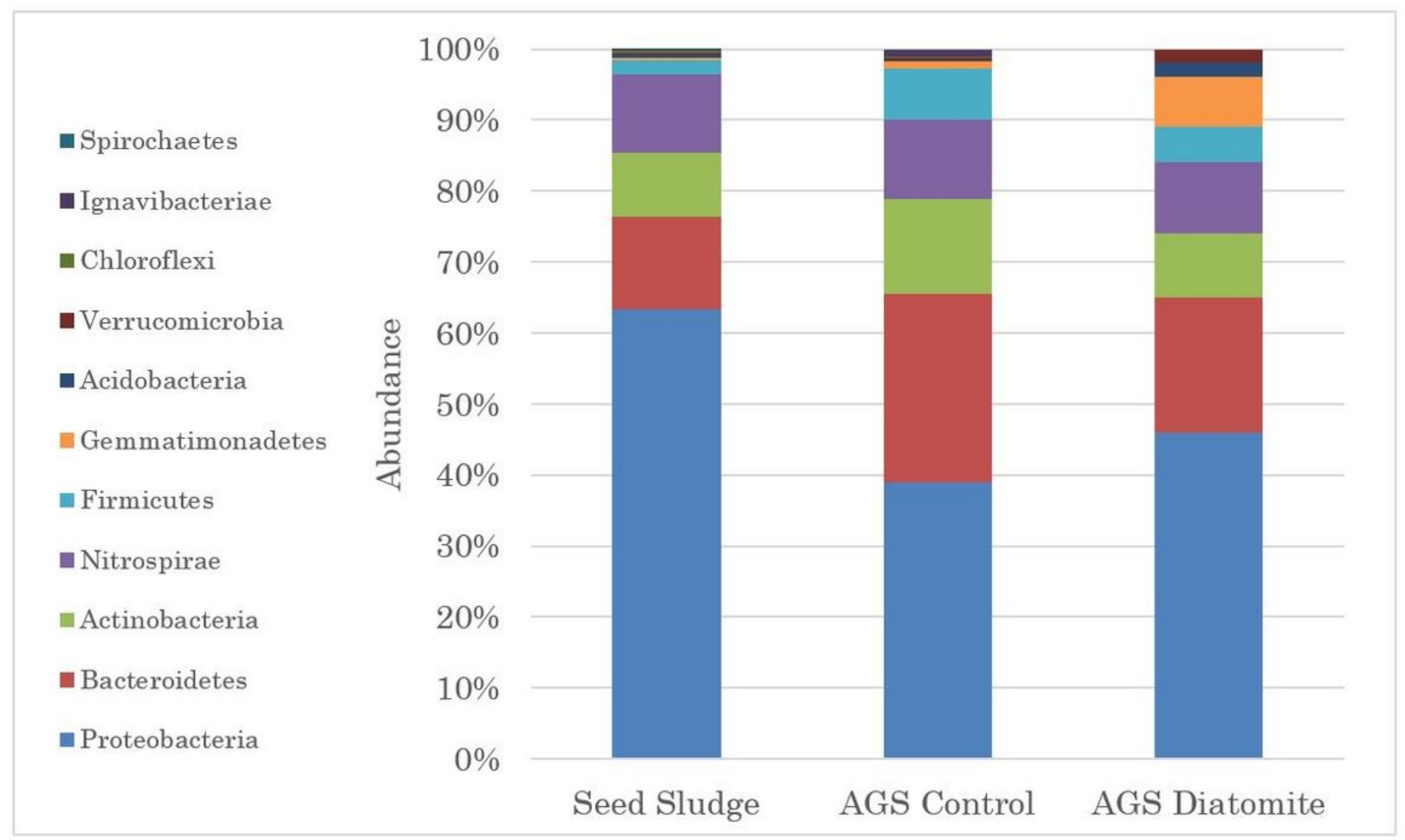

\section{Figure 2}

Phylum level of bacteria community of seed sludge, AGS control and AGS diatomite 


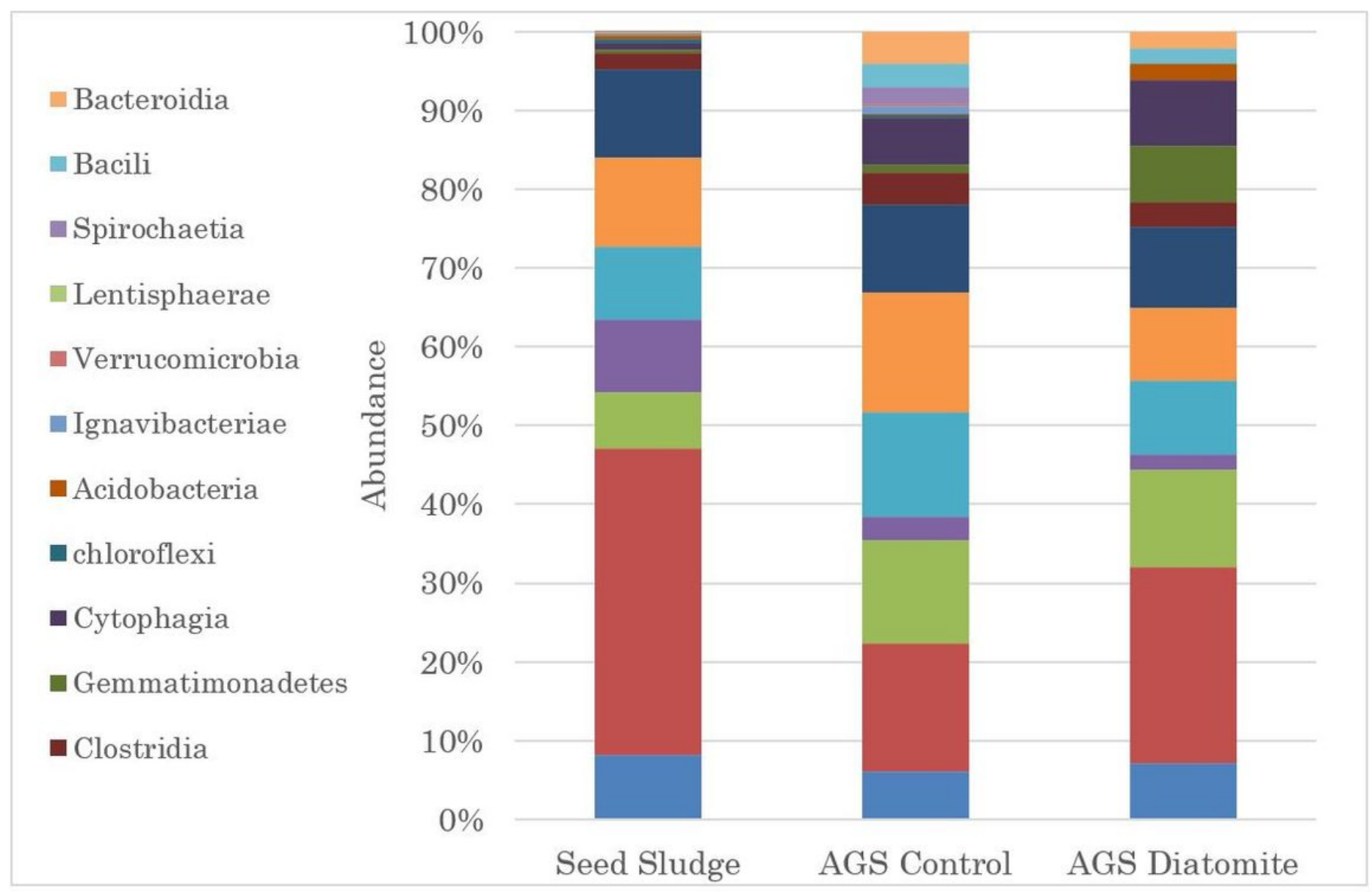

Figure 3

Class level of bacteria community of seed sludge, AGS control and AGS diatomite 